OPEN ACCESS

Edited by:

Jaime J. Carvajal,

Andalusian Center for Development Biology (CABD), Spain

Reviewed by:

Piero Crespo,

Consejo Superior de Investigaciones

Científicas, Spain

Tamer Saad Kaoud,

University of Texas at Austin

United States

*Correspondence:

Beom-Jun Kim

umkbj0825@amc.seoul.kr

${ }^{\dagger}$ These authors have contributed equally to this work

Specialty section:

This article was submitted to

Molecular Medicine,

a section of the journal

Frontiers in Cell and Developmental

Biology

Received: 26 May 2020 Accepted: 21 October 2020 Published: 06 November 2020

Citation:

Lee JY, Park SJ, Kim DA, Lee SH,

Koh J-M and Kim B-J (2020)

Muscle-Derived Lumican Stimulates Bone Formation via Integrin $\alpha 2 \beta 1$ and the Downstream ERK Signal. Front. Cell Dev. Biol. 8:565826. doi: 10.3389/fcell.2020.565826

\section{Muscle-Derived Lumican Stimulates Bone Formation via Integrin $\alpha 2 \beta 1$ and the Downstream ERK Signal}

\author{
Jin Young Lee ${ }^{1 \dagger}$, So Jeong Park' ${ }^{1 \dagger}$, Da Ae Kim', Seung Hun Lee ${ }^{2}$, Jung-Min Koh² and \\ Beom-Jun Kim ${ }^{2 *}$ \\ ${ }^{1}$ Asan Institute for Life Sciences, Asan Medical Center, University of Ulsan College of Medicine, Seoul, South Korea, \\ ${ }^{2}$ Division of Endocrinology and Metabolism, Asan Medical Center, University of Ulsan College of Medicine, Seoul, \\ South Korea
}

Skeletal muscle and bone are highly interrelated, and previous proteomic analyses suggest that lumican is one of muscle-derived factors. To further understand the role of lumican as a myokine affecting adjacent bone metabolism, we investigated the effects of lumican on osteoblast biology. Lumican expression was significantly higher in the cell lysates and conditioned media (CM) of myotubes than those of undifferentiated myoblasts, and the known anabolic effects of myotube CM on osteoblasts were reduced by excluding lumican from the $\mathrm{CM}$. Lumican stimulated preosteoblast viability and differentiation, resulting in increased calvaria bone formation. The expression of osteoblast differentiation markers was consistently increased by lumican. Lumican increased the phosphorylation of ERK, whereas ERK inhibitors completely reversed lumican-mediated stimulation of Runx2 and ALP activities in osteoblasts. Results of a binding ELISA experiment in osteoblasts show that transmembrane integrin $\alpha 2 \beta 1$ directly interacted with lumican, and an integrin $\alpha 2 \beta 1$ inhibitor attenuated the stimulation of ERK and ALP activities by lumican. Taken together, the results indicate that musclederived lumican stimulates bone formation via integrin $\alpha 2 \beta 1$ and the downstream ERK signal, indicating that this is a potential therapeutic target for metabolic bone diseases.

Keywords: lumican, osteoblast, bone formation, integrin $\alpha 2 \beta 1$, ERK

\section{INTRODUCTION}

Bone and skeletal muscle contribute the largest amount of tissues in a lean individual; both respond to physical activity and play a role in protecting internal organs (Tagliaferri et al., 2015). Accumulating clinical evidence indicates that bone and muscle health are highly interrelated, working together throughout an individual's lifetime (Hirschfeld et al., 2017; Bettis et al., 2018). For example, concomitant losses in bone and muscle mass are frequently observed in older adults (Verschueren et al., 2013; Kim et al., 2015). Both osteoporosis and sarcopenia contribute to the development of fragility fractures that contribute to high disability and mortality (Yu et al., 2014). Thus, effective measures to prevent fractures require continuous efforts to understand the mechanisms underlying bone-muscle crosstalk.

Traditionally, the parallel changes of bone and muscle have been explained by the mechanical force transduction generated by muscle contraction to the adjacent bone (Burr et al., 2002; 
Qin et al., 2010; Hong and Kim, 2018). Interestingly, aside from being a component of the locomotor system, skeletal muscle is acknowledged as a secretory organ (Pedersen and Febbraio, 2012), and thus it has been hypothesized that muscle-derived factors called "myokines" act as a paracrine signal to regulate bone homeostasis (Hamrick et al., 2010; Kaji, 2014; Giudice and Taylor, 2017). This possibility has been supported in a murine model showing that muscle flaps, compared with other tissues, effectively accelerates fracture healing (Harry et al., 2008, 2009). In addition, recent experiments show that conditioned media (CM) collected from myotubes significantly increase bone formation, suggesting that muscles play a dominant role in releasing bone anabolic factors (Lee et al., 2019).

Evidence is increasing for the importance of biochemical communication between skeletal muscle and bones as well as with other organs, such as brain, pancreas, liver, and adipose tissue (Giudice and Taylor, 2017; Romagnoli et al., 2019). This has led to efforts to identify specific muscle-secreting factors. For example, Norheim et al. (2011) performed, via database searches, proteomic analyses on the CM from cultured human myotubes, and identified 17 novel proteins with secretory features. Among these factors, we are particularly interested in lumican, because it has been consistently detected in the myotube CM of all donors and its expression is markedly increased in skeletal muscle after strength training (Norheim et al., 2011). Importantly, several experiments indicate that lumican may affect the integrin and ERK signaling (Seomun and Joo, 2008; Brezillon et al., 2013), which are key pathways in bone metabolism. These backgrounds raise the possibility that lumican could act as a myokine on adjacent bone homeostasis. To examine this hypothesis, we tested its effects on osteoblast biology through in vitro and animal experiments.

\section{MATERIALS AND METHODS}

\section{Cell Culture}

Mouse C2C12 myoblasts were purchased from the American Type Culture Collection (ATCC, Manassas, VA, United States) and maintained in DMEM supplemented with 10\% FBS, $20 \mathrm{mM}$ HEPES, 2 mM L-glutamine, and antibiotics (Life Technologies Corp., Carlsbad, CA, United States) at $37^{\circ} \mathrm{C}$ in a humidified atmosphere containing $5 \% \mathrm{CO}_{2}$. CM collected after further incubation for $24 \mathrm{~h}$ in serum- and phenol red-free media was regarded as myoblast CM. To induce myogenic differentiation, cells were grown to $90 \%$ confluency in maintenance media and then switched to differentiation media (DMEM with $2 \%$ horse serum) and cultured for 3 days. Myotube CM was again collected after further incubation for $24 \mathrm{~h}$ in serum- and phenol red-free media (Lee et al., 2019). All collected CM was filtered through a $0.45 \mu \mathrm{m}$ membrane filter and precipitated by lyophilization.

Murine preosteoblast MC3T3-E1 cells (ATCC) were cultured at $37^{\circ} \mathrm{C}$ in $\alpha$-minimum essential medium ( $\alpha$-MEM) containing $10 \% \mathrm{FBS}, 100 \mathrm{U} / \mathrm{mL}$ penicillin, and $100 \mu \mathrm{g} / \mathrm{mL}$ streptomycin in a humidified atmosphere with $5 \% \mathrm{CO}_{2}$. The media was changed every 2-3 days. Upon reaching $80 \%$ confluence, cells were subcultured with trypsin-EDTA (Gibco, Grand Island, NY,
United States). The cells were differentiated into osteoblasts with $50 \mu \mathrm{g} / \mathrm{mL}$ ascorbic acid and $10 \mathrm{mM} \beta$-glycerophosphate (SigmaAldrich, St. Louis, MO, United States) for 7 days. Human fetal osteoblastic (hFOB) cells were cultured at $33^{\circ} \mathrm{C}$ in $1: 1$ mixture of Ham's F12 Medium Dulbecco's Modified Eagle's Medium (DME/F-12 1:1, Hyclone, Logan, UT, United States) containing $10 \% \mathrm{FBS}, 100 \mathrm{U} / \mathrm{mL}$ penicillin, and $100 \mu \mathrm{g} / \mathrm{mL}$ streptomycin in a humidified atmosphere with $5 \% \mathrm{CO}_{2}$.

\section{Western Blot Analysis}

Cells were lysed in RIPA buffer [50 mM Tris-HCl ( $\mathrm{pH} 7.4$ ), $150 \mathrm{mM} \mathrm{NaCl}, 1 \%$ Triton X-100, 1 mM EDTA, 1 mM EGTA, $0.1 \%$ SDS, $1 \%$ sodium deoxycholate, $1 \mathrm{mM} \mathrm{Na3VO} 4,1 \mathrm{mM} \mathrm{NaF}$, $1 \mathrm{mM}$ PMSF, and protease inhibitor cocktail]. After a 30-min incubation on ice, lysates were centrifuged at $14,000 \mathrm{rpm}$ for $20 \mathrm{~min}$ at $4^{\circ} \mathrm{C}$. The protein concentration was measured with a BCA protein assay kit (Pierce Chemical Co., Rockford, IL, United States). Protein samples were separated by SDS-PAGE and transferred to a polyvinylidene fluoride (PVDF) membrane followed by immunoblotting with antibodies (Park et al., 2019). The primary antibodies are as follows: lumican (ab168348) and myogenin (Myog; ab1845) purchased from Abcam (Cambridge, MA, United States); phospho-ERK (9101), phospho-JNK (9251), phospho-AKT (9271), phospho-FAK (Tyr397, 3283), phosphorSrc (Tyr416, 2101), ERK (9102), JNK (9252), AKT (9272), SMAD4 (38454), and integrin $\beta 1$ (34971) purchased from Cell Signaling Technology (Beverly, MA, United States); integrin $\alpha 2$ (sc-74466); and troponin-C (sc-48347) purchased from Santa Cruz Biotechnology (Dallas, TX, United States); $\alpha$-tubulin (T9026), $\beta$-actin (A3894), and myosin heavy chain (MyHC; M1570) purchased from Sigma-Aldrich.

\section{Immunofluorescence}

C2C12 myoblasts and myotubes were fixed in $4 \%$ paraformaldehyde (PFA) for $10 \mathrm{~min}$, washed twice with PBS, permeabilized in $0.01 \mathrm{M}$ sodium citrate buffer containing $0.1 \%$ Triton X-100 for $10 \mathrm{~min}$, and washed twice with PBS (Kim et al., 2018). Next, cells were blocked with $2 \%$ bovine serum albumin (BSA) in PBS for 1 h. Primary antibodies, such as anti-MyHC (MF20; Developmental Studies Hybridoma Bank, Iowa City, IA, United States) and anti-lumican (Abcam, 1:1000 dilution), were incubated at $4^{\circ} \mathrm{C}$ overnight. Cells were incubated with Alexa Flour 555-conjugated secondary antibodies (Cell Signaling, 1:1000 dilution) for $1 \mathrm{~h}$, then washed with PBST (0.1\% Tween-20 in PBS). Then, the cells were incubated with $4^{\prime}, 6^{\prime}$-diamidino-2-phenyindole (DAPI; Sigma-Aldrich, 1:10000 dilution) for $2 \mathrm{~min}$ and washed with PBS. The samples were mounted using Fluoromount G (Southern Biotech, Birmingham, AL, United States) and images were obtained using a fluorescence microscope (Carl Zeiss, Jena, Germany).

The hFOB cells were washed three times with PBS after treatment with $40 \mathrm{nM} 6 \mathrm{X}$ His-tagged lumican (R\&D systems Inc., Minneapolis, MN, United States) for $30 \mathrm{~min}$. Then cells were fixed with 4\% PFA for $10 \mathrm{~min}$, permeabilized with $0.1 \%$ Triton X-100 in PBS for $10 \mathrm{~min}$ at room temperature. After blocking with $1 \%$ BSA in PBS for $60 \mathrm{~min}$, cells were incubated 
with anti-6X His (Abcam) and anti-integrin $\alpha 2$ and $\beta 1$ (Abcam) for $16 \mathrm{~h}$ at $4^{\circ} \mathrm{C}$. Cells were washed with PBS and incubated with Alexa Fluor 488-conjugated and Alexa Fluor 555-conjugated secondary antibodies for $1 \mathrm{~h}$ at room temperature. Nuclei were counterstained with DAPI. The stained cells were mounted, and images were obtained using an LSM 710 confocal laser scanning microscope (Carl Zeiss).

\section{Quantitative Reverse-Transcription PCR}

Total RNA was extracted using TRIzol reagent (Invitrogen, Carlsbad, CA, United States) according to the manufacturer's protocol. After first-strand cDNA synthesis with the Superscript III First-Strand Synthesis System (Invitrogen) using oligo dT primers, Quantitative Reverse-Transcription PCR (qRT-PCR) was performed in triplicate on a Light Cycler ${ }^{\circledR} 480$ SYBR Green I Master (Roche, Mannheim, Germany; Kim et al., 2018). The primers for lumican (NM_008524), Myog (NM_031189), Runtrelated transcription factor 2 (Runx2; NM_001146038), osterix (Osx; AY803733), collagen type $1 \alpha$ (Col1 $\alpha$; NM_007742), alkaline phosphatase (Alp; NM_007431), osteocalcin (Ocn; NM_007541), integrin $\alpha 2$ (Itg $\alpha 2 ;$ NM_008396.3), and integrin $\beta 1$ (Itg $\beta 1$; NM_010578) were obtained from Applied Biosystems (Foster City, CA, United States). The threshold cycle (Ct) value for each gene was normalized to the $\mathrm{Ct}$ value of the $18 \mathrm{~S}$ rRNA (NR_003278.3).

\section{Lumican Silencing Using Short Hairpin RNA}

The mouse lumican short hairpin RNA (shRNA; 5'-CCT GGA AAC TCG TTT AAT ATA-3') was constructed using the pLKO.1lumican shRNA cloning vector (Sigma-Aldrich). To knockdown lumican, $70-80 \%$ confluent $\mathrm{C} 2 \mathrm{C} 12$ cells were infected with control lumican shRNA CM or control shRNA CM in the presence of $8 \mu \mathrm{g} / \mathrm{mL}$ polybrene for $6 \mathrm{~h}$. The cells were then washed with PBS and placed in growth media. After incubation for $48 \mathrm{~h}$, infected myoblasts were differentiated into myotubes, and the CM was collected in the same manner as described above.

\section{Viability Assay}

Cell viability was measured using the Cell Counting Kit8 (CCK-8; Dojindo, Kumamoto, Japan) according to the manufacturer's instructions. Briefly, $10 \mu \mathrm{L}$ of WST-8 dye [2-(2-methoxy-4-nitrophenyl)-3-(4-nitrophenyl)-5-(2,4disulfophenyl)-2H-tetrazolium, monosodium salt] was added to each well of a 96-well plate (Lee et al., 2020). The suspension was incubated for $1 \mathrm{~h}$, and the absorbance was then read at $450 \mathrm{~nm}$ with a reference wavelength of $650 \mathrm{~nm}$ using a microplate reader (SPECTRAmax 340PC; Molecular Devices, Palo Alto, CA, United States).

\section{Migration Assay}

The chemotaxis assay was performed in a Boyden chamber system using a transwell with an 8 - $\mu \mathrm{m}$-pore size polycarbonate membrane (Corning, NY, United States). Cells were seeded onto the inner chamber at a density of $1.0 \times 10^{5}$ cells $/ 100 \mu \mathrm{L}$ in $\alpha$-MEM with FBS for $6 \mathrm{~h}$ and then exposed to lumican in the outer chamber for an additional $24 \mathrm{~h}$ (Park et al., 2019). The cells on the upper membrane were then completely removed by wiping with a cotton swab, while cells on the lower surface of the membrane were fixed in 4\% PFA, stained with crystal violet, photographed, and counted under a dissecting microscope (Carl Zeiss).

\section{ALP Activity and Osteocalcin Secretion Assays}

MC3T3-E1 cells were seeded in 12-well plates at a density of $5 \times 10^{4}$ cells/well and then allowed to differentiate into osteoblasts for 7 days. Cells were then washed with PBS, and ALP activity was measured using the p-nitrophenyl phosphate hydrolysis method (Sabokbar et al., 1994). ALP activity was normalized relative to the corresponding total cellular protein content, which was determined by the BCA assay (Pierce Chemical Co.). Osteocalcin concentration in the culture media was measured using an osteocalcin ELISA kit (BT-470; Alfa Aesar, Ward Hill, MA, United States; Sabokbar et al., 1994).

\section{Luciferase Activity Assay}

MC3T3-E1 cells were seeded in 24-well plates at a density of $3 \times 10^{4}$ cells/well and cells were transfected with $100 \mathrm{ng}$ of pGL3 vector and $100 \mathrm{ng}$ of Runx2 (6xOSE) luciferase reporter plasmid using Lipofectamine 2000 (Invitrogen; Kim et al., 2018). After $6 \mathrm{~h}$, transfected cells were washed and growth medium was added to the plate. The next day, cells were treated with lumican or ERK inhibitors (PD98059 and U0126; Cell Signaling Technology) and allowed to differentiate into osteoblasts. After 3 days, cells were washed with PBS and lysed in $150 \mu l$ of passive lysis buffer. Activation of Runx2 was confirmed using a Dual-Luciferase ${ }^{\circledR}$ Reporter Assay System (Promega, Madison, WI, United States), and luciferase activity was measured using a microplate luminometer (MicroLumat Plus LB96V; Berthold Technologies, Oak Ridge, TN, United States). Relative activity was calculated as the ratio of the firefly reporter to the Renilla luciferase control (pRL-SV40).

\section{Ligand and Receptor Binding Assay}

Lumican was coated onto the wells of Maxisorp 96-well microtiter plates and incubated for $18 \mathrm{~h}$ at $4^{\circ} \mathrm{C}$. Each well was washed three times with PBST (0.2\% Tween-20 in PBS), then the plates were blocked with $1 \%$ BSA in PBST for $2 \mathrm{~h}$. Cell lysates were added to the plates, incubated for $2 \mathrm{~h}$, then the wells were washed three times (Kim et al., 2018; Park et al., 2019). Preparations of integrin $\alpha 2$ (Santa Cruz Biotechnology) or $\beta 1$ antibody (Cell Signaling Technology) in blocking solution were added to the plates and allowed to react for $2 \mathrm{~h}$. After washing, HRP-linked antibody (Cell signaling Technology) was added and the lysates were incubated for $2 \mathrm{~h}$, and then washed five times. The reaction was developed with $100 \mu \mathrm{l}$ TMB substrate solution and stopped with $100 \mu \mathrm{l}$ of $1 \mathrm{~N} \mathrm{H}_{2} \mathrm{SO}_{4}$. Microtiter plates were measured at $450 \mathrm{~nm}$ using a microplate reader (Infinite $200 \mathrm{PRO}$, Tecan Life Sciences, Zurich, Switzerland). 


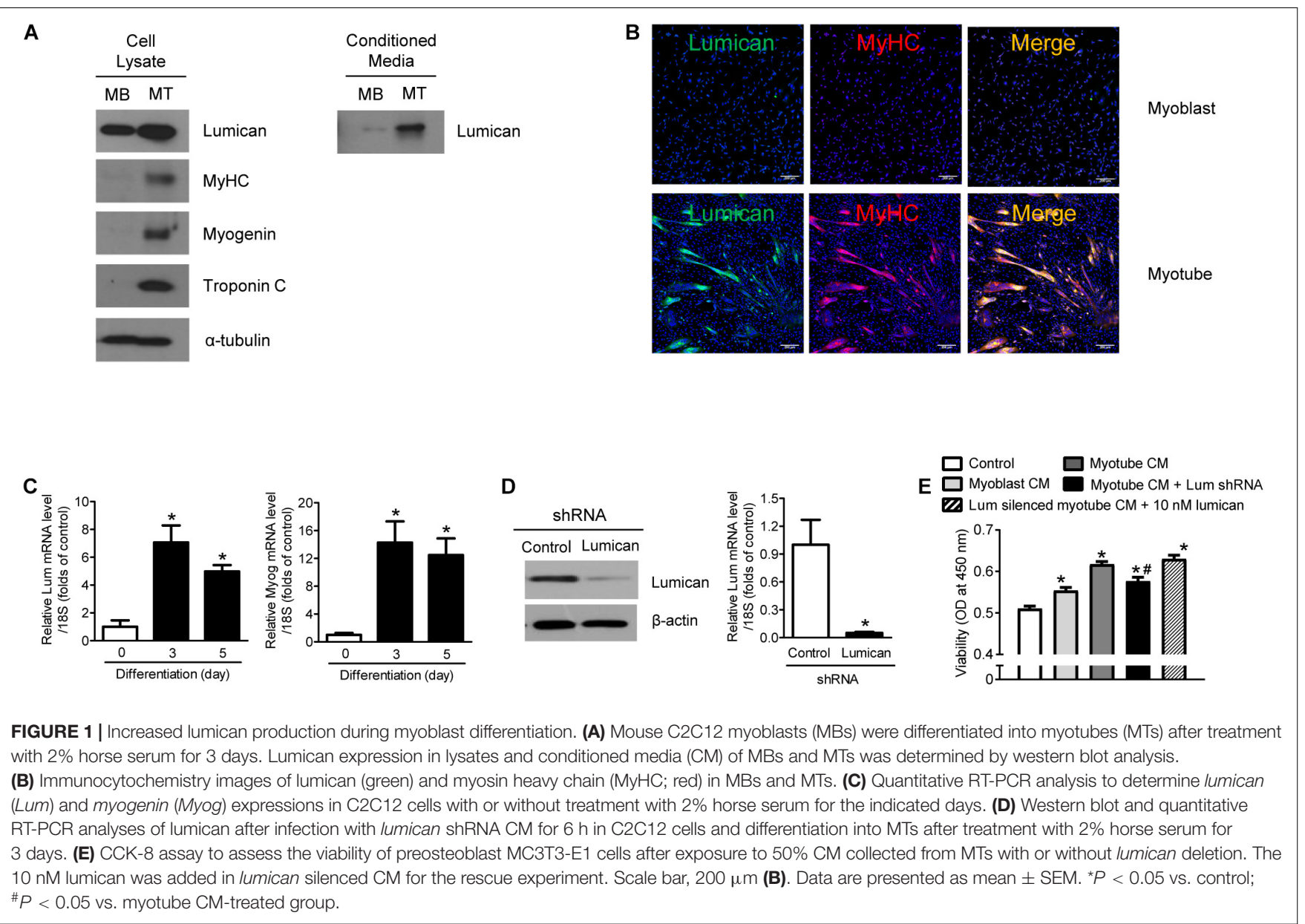

\section{In vivo Calvaria Bone Formation}

Five-week-old male C57BL/6 mice (Orientbio, Seongnam, South Korea) were used in this study. Recombinant lumican (200 $\mu \mathrm{g} / \mathrm{kg}$ ) or PBS was subcutaneously injected with a 31gage needle into the right or left parietal bones, respectively, 3 times a week for 4 weeks. Mice were sacrificed 1 week following treatment, and calvaria bones were fixed in $4 \%$ PFA for $24 \mathrm{~h}$ and decalcified in $0.5 \mathrm{M}$ EDTA in PBS for 2-4 weeks. Decalcified specimens were embedded in paraffin and then coronally sectioned at a thickness of $6 \mu \mathrm{m}$. After deparaffinization, sections were rehydrated and then stained with either hematoxylin and eosin (H\&E, Sigma-Aldrich) following the manufacturer's instructions. Using Image-Pro Plus software (Media Cybernetics, Rockville, MD, United States), bone widths were measured at 5 adjacent locations that were at the same distance from the midline between the sagittal suture and the site of muscle attachment, and their mean values were calculated (Lee et al., 2020). To count the number of osteoblasts, the rehydrated sections were stained with toluidine blue $\mathrm{O}$ solution (Sigma-Aldrich), and images of the stained osteoblasts were captured using cellSens Standard BX53 software (Olympus, Tokyo, Japan). All methods for animal care and experimental procedures were reviewed and approved by the Institutional Animal Care and Use Committee of the Asan Institute for Life
Sciences. The committee abides by the institute of Laboratory Animal Resources (ILAR) guide. All experiments were done, according with the Korean Ministry of Food and Drug Safety (MFDS) guidelines.

\section{Ex vivo Culture}

The calvaria bone of timed-pregnant ICR mice (Orientbio) at E21.0 was cut in half along the calvaria sagittal line to include the frontal, coronal, and lambdoid sutures. The bone was placed in a transwell with an 8 - $\mu \mathrm{m}$-pore size polycarbonate membrane. To silence lumican expression, each calvaria bone was infected with lumican shRNA CM or control shRNA CM in BGjb medium (Gibco) containing $100 \mathrm{U} / \mathrm{mL}$ penicillin, and $100 \mu \mathrm{g} / \mathrm{mL}$ streptomycin and $0.1 \% \mathrm{BSA}$ in the presence of $8 \mu \mathrm{g} / \mathrm{mL}$ polybrene. After 3 days, each bone was cultured for an additional 10 days in a medium containing $50 \mu \mathrm{g} / \mathrm{mL}$ of insulin (Sigma-Aldrich). The bone then was fixed in 4\% PFA for $24 \mathrm{~h}$ and decalcified in $0.5 \mathrm{M}$ EDTA in PBS for 2 days. The calvaria specimens were embedded in paraffin, coronally sectioned at a thickness of $3 \mu \mathrm{m}$, and stained with $\mathrm{H} \& \mathrm{E}$ or toluidine blue $\mathrm{O}$ solution (Marino et al., 2016). Calvaria bone widths and the number of osteoblasts were assessed using the same methods described above. 
A

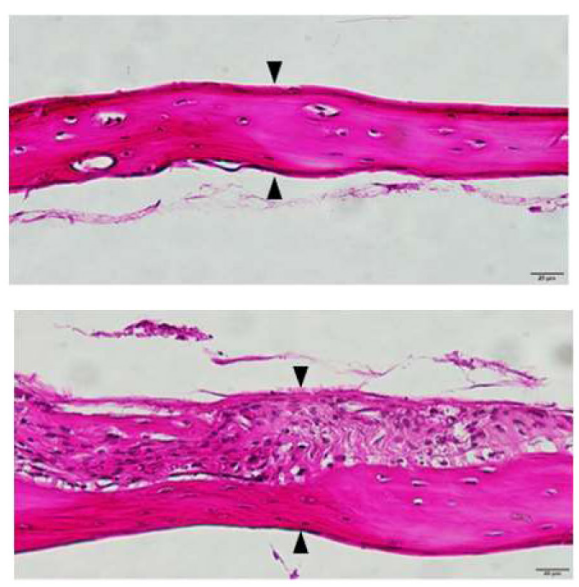

B

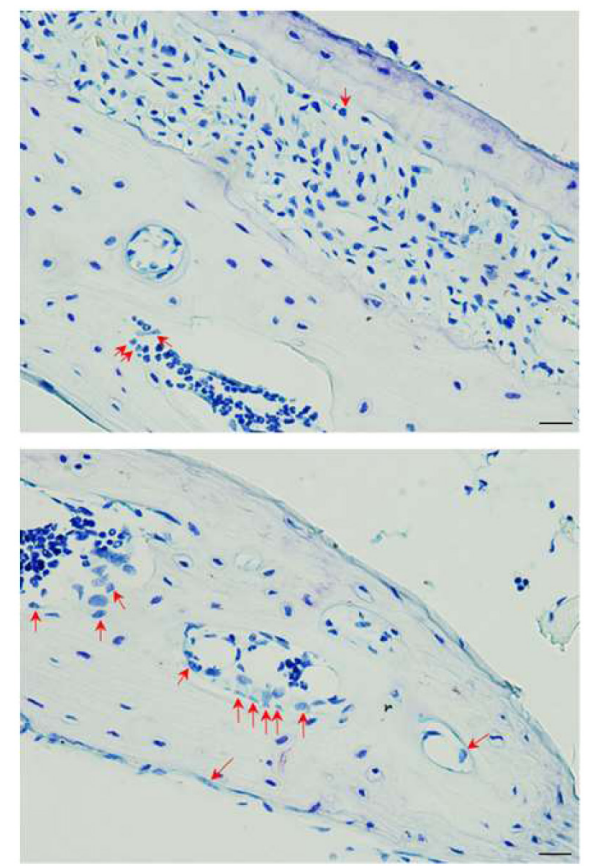

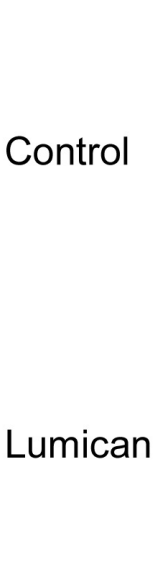

Control
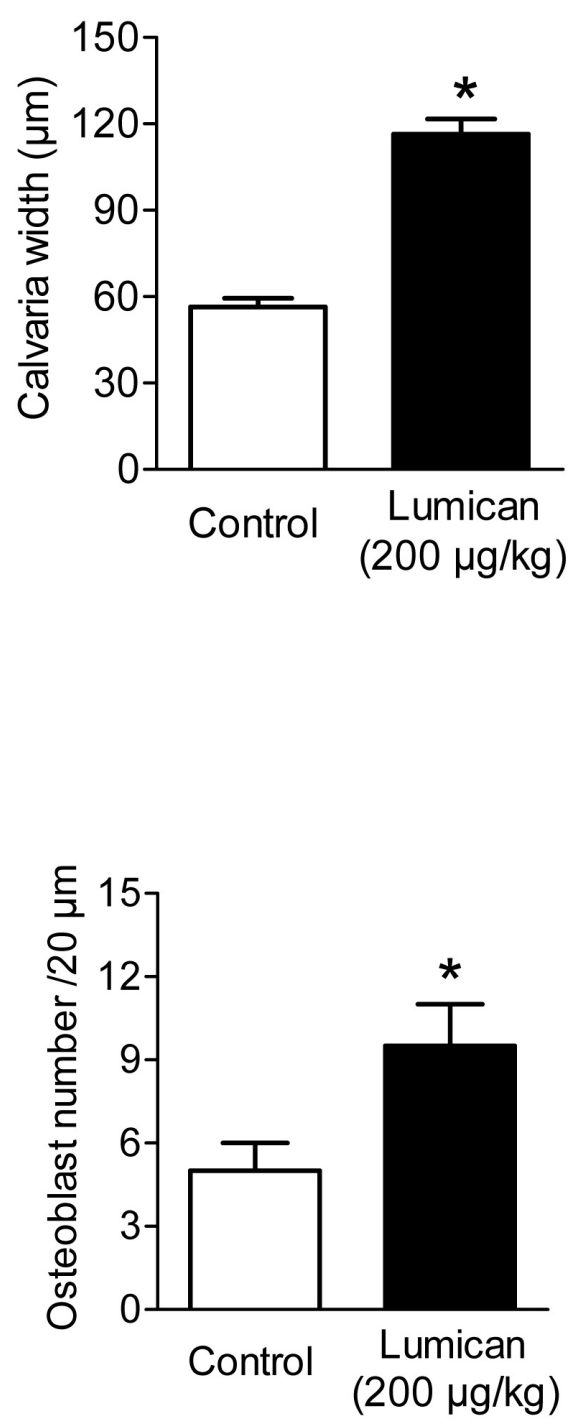

FIGURE 2 | Increase in calvaria bone formation by lumican. Recombinant lumican was injected into the right side of the calvaria of 5-week-old male C57BL/6 mice for 4 weeks. PBS was injected into the left side of the calvaria as a negative control. (A) Hematoxylin and eosin staining was performed on the calvaria bone sections. Calvaria bone widths were quantitated by analyzing the average widths of five spots at the same interval of the midline between the sagittal suture and the site of muscle attachment. Black arrows indicate osteoid lines as reference for calvaria bone width measurements. (B) Toluidine blue O staining was also performed. Red arrows indicate osteoblasts, which are mono nuclear cuboidal cells on bone surface (B). Scale bars, $20 \mu \mathrm{m}$ (A,B). Data are presented as mean \pm SEM. ${ }^{\star} P<0.05$ vs. control.

\section{Statistical Analysis}

Unless otherwise specified, all data are expressed as the mean \pm standard error of the mean of at least three independent experiments relying on triplicate measurements. The significance of differences between two groups was assessed using the Mann-Whitney $U$ test, whereas differences between 3 or more groups were tested using the analysis of variance with posthoc analysis via Tukey's honest significance test (Kim et al., 2019). All statistical analyses were performed using SPSS version 18.0 (SPSS,
Inc., Chicago, IL, United States). $P<0.05$ was considered statistically significant.

\section{RESULTS}

\section{Increased Lumican Production During Myoblast Differentiation}

Western blot analysis reveals that lumican expression was higher in the cell lysates and CM of myotubes differentiated by $2 \%$ horse 

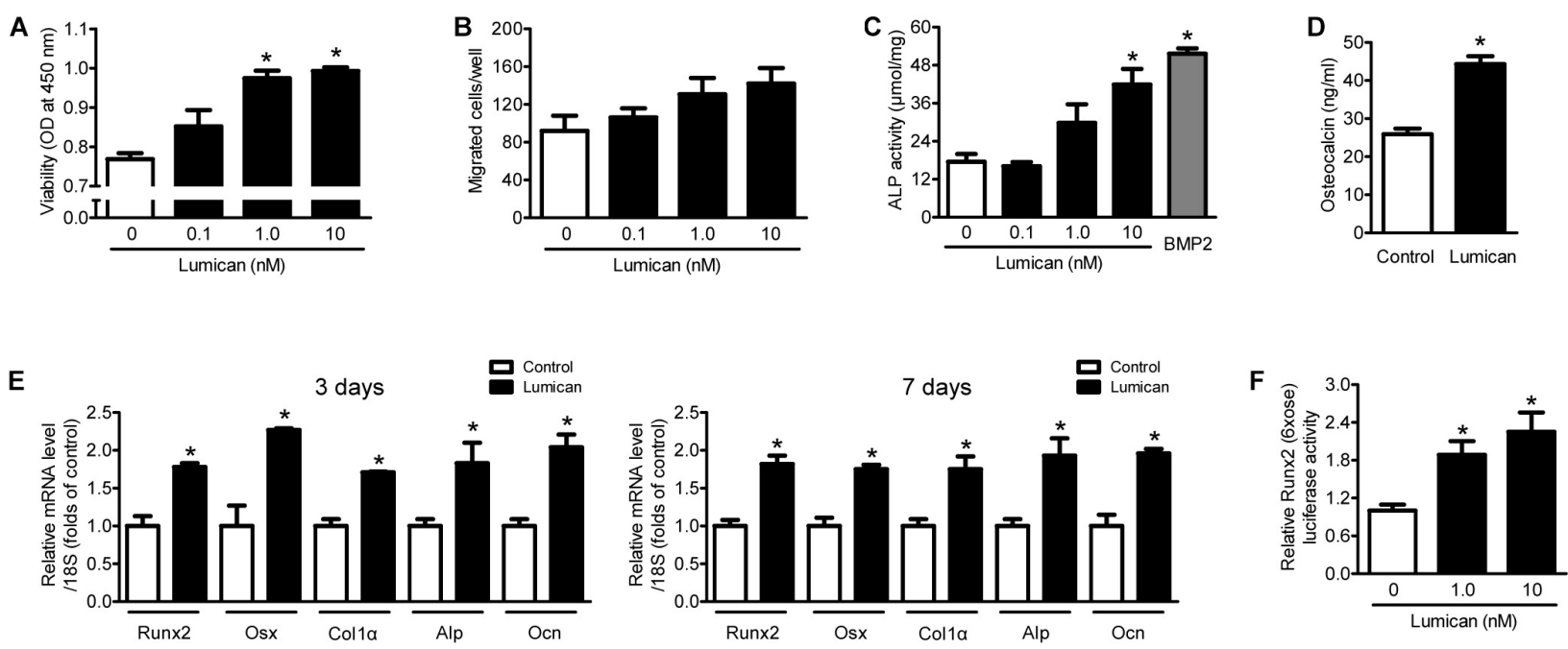

FIGURE 3 | Lumican stimulates preosteoblast viability and differentiation. (A) The viability and (B) directional migration of preosteoblast MC3T3-E1 cells were assessed by CCK-8 assay and a Boyden chamber system, respectively, after exposure to the indicated concentrations of lumican for 24 h. (C) Alkaline phosphatase (ALP) activity and (D) osteocalcin secretion of MC3T3-E1 cells in osteogenic medium containing $50 \mu \mathrm{g} / \mathrm{mL}$ ascorbic acid and $10 \mathrm{mM} \beta$-glycerophosphate with or without $10 \mathrm{nM}$ lumican for 7 days. ALP activity was normalized by total cellular protein amounts. (E) Quantitative RT-PCR expression analysis of osteoblast differentiation markers in MC3T3-E1 cells cultured in osteogenic medium with or without 10 nM lumican for 3 or 7 days. (F) Luciferase activity of Runx2 after exposure to the indicated concentrations of lumican in osteogenic medium for 3 days. Data are presented as mean \pm SEM. ${ }^{\star} P<0.05$ vs. control.

serum from mouse C2C12 myoblasts than in cell lysates and CM from undifferentiated cells (Figure 1A). Immunocytochemistry and qRT-PCR analyses further confirms that lumican expression was significantly higher in mature myotubes than in myoblasts (Figures 1B,C, respectively). A previous study has demonstrated that myotube CM markedly stimulates preosteoblast viability, compared to non-CM (i.e., control), and myoblast CM (Lee et al., 2019). To determine whether lumican acts as a myokine that affects bone metabolism, C2C12 myoblasts infected with lumican shRNA CM were differentiated into myotubes in the presence of $2 \%$ horse serum (Figure 1D), and their CM was collected. Interestingly, the stimulation of preosteoblast viability by myotube $\mathrm{CM}$ was markedly diminished by lumican silencing, and the addition of lumican to these CM rescued the reduced preosteoblast viability resulting from lumican knockdown (Figure 1E). Taken together, these results imply that lumican secreted from mature myotubes could function positively in osteoblast biology.

\section{Lumican Increases Bone Formation Through the Stimulation of Preosteoblast Viability and Differentiation}

To investigate the effects on in vivo bone formation, recombinant lumican and PBS were injected into the right and left sides of the mouse calvaria bone, respectively. Lumican treatment increased calvaria thickness by 2.1-fold, compared to the PBStreated control (Figure 2A), and more osteoblasts were observed on the lumican-treated calvaria bone surface than in the control (Figure 2B).

To supplement the mouse findings described above, we treated murine preosteoblast MC3T3-E1 cells with lumican in vitro. Lumican stimulated preosteoblast viability in a dosedependent manner (Figure 3A), while preosteoblast migration changed little following lumican treatment (Figure 3B). Lumican markedly increased osteoblast differentiation, as determined by ALP activity (Figure 3C) and Ocn secretion (Figure 3D). Consistently, the expression levels of Runx2 and Osx, and their target genes involved in osteoblast differentiation, including Coll $\alpha, A l p$, and Ocn, were significantly increased by lumican (Figure 3E). Upregulation of Runx2 was also confirmed by luciferase assays (Figure 3F). Collectively, these data indicate that lumican contributes to bone formation through the stimulation of preosteoblast viability and differentiation.

\section{The Effects of Lumican on Osteoblastogenesis Are Mediated by the Stimulation of the ERK Signal}

To determine the mechanism of action of lumican on osteoblasts, we focused on several signaling pathways related to osteoblast differentiation. Western blot analyses show that lumican increased the activity of ERK, but not those of JNK, Akt, and SMAD4 (Figure 4A). Importantly, pretreatment with the ERK inhibitors, PD98059 and U0126, almost completely reversed the lumican-mediated stimulation of Runx2 and ALP activities (Figures 4B,C, respectively), indicating that ERK is a key signal regulating the effect of lumican on osteoblastogenesis.

\section{Integrin $\alpha 2 \beta 1$ Is the Major Receptor for Lumican in Osteoblasts}

Transmembrane integrin $\alpha 2 \beta 1$ is known to be involved in the survival and differentiation of osteoblast lineages (Popov et al., 2011; Shih et al., 2011) as well as in the action of lumican in 

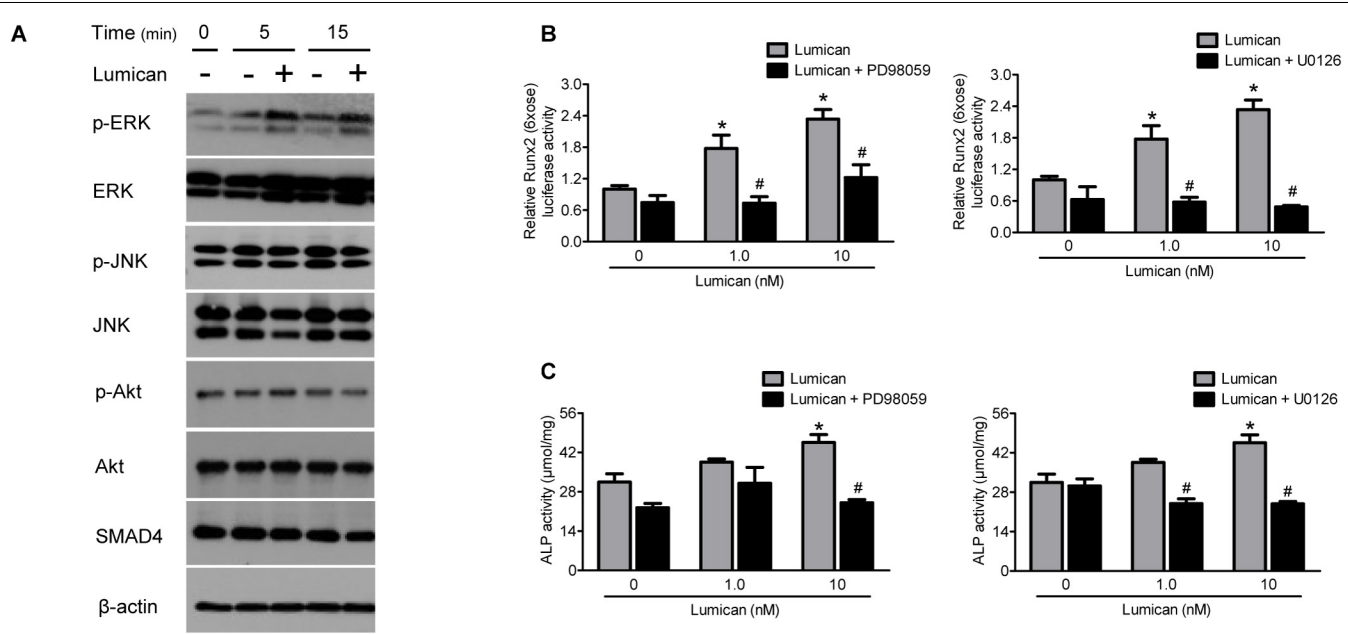

FIGURE 4 | Effects of lumican on osteoblastogenesis are mediated by the simulation of ERK signal. (A) Western blot analysis to determine the activity of signals related to osteoblast differentiation after treatment with $10 \mathrm{nM}$ lumican in MC3T3-E1 cells. (B,C) Murine preosteoblast MC3T3-E1 cells were pretreated with $20 \mu \mathrm{M}$ PD98059 or $10 \mu \mathrm{M} \cup 0126$, ERK inhibitors, followed by treatment with the indicated concentrations of lumican in the presence of $50 \mu \mathrm{g} / \mathrm{mL}$ ascorbic acid and $10 \mathrm{mM} \beta$-glycerophosphate. Runx2 and ALP activities were assessed after 5 and 7 days, respectively. Data are presented as mean \pm SEM. ${ }^{\star} P<0.05$ vs. control; ${ }^{\#} P<0.05$ vs. lumican-treated group.
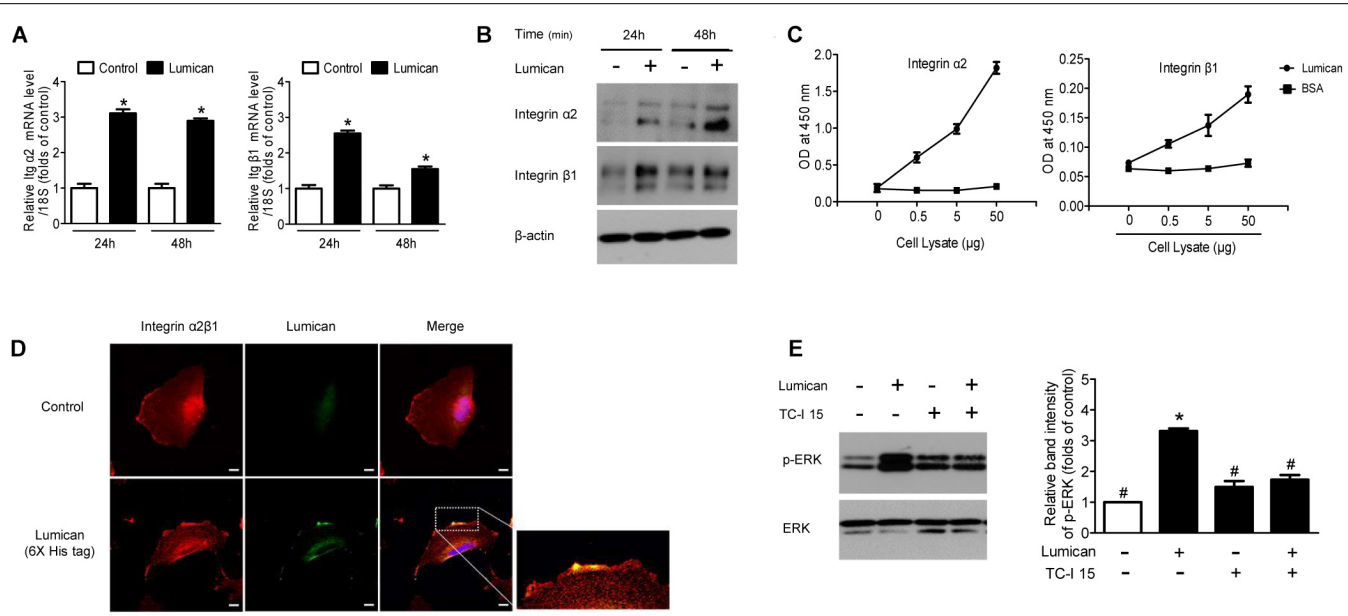

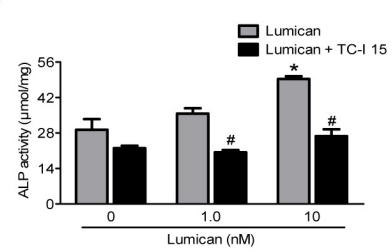

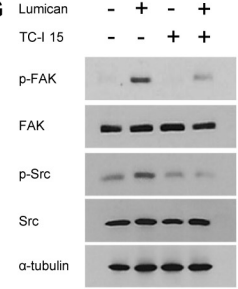
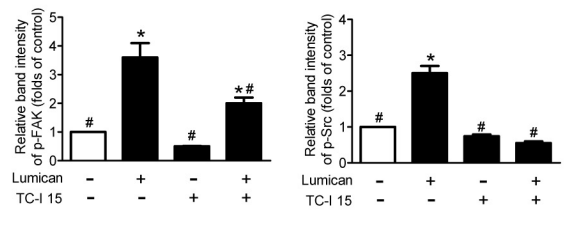

FIGURE 5 | Integrin $\alpha 2 \beta 1$ is the major receptor for lumican on osteoblasts. (A,B) Quantitative RT-PCR and western blot analyses to determine the activity of integrin $\alpha 2$ and $\beta 1$ after treatment with $10 \mathrm{nM}$ lumican in MC3T3-E1 cells, respectively. (C) Interaction of lumican with integrin $\alpha 2$ and $\beta 1$ in MC3T3-E1 cells using a binding ELSIA assay. Different amounts of cell lysates were incubated in lumican- or BSA-coated wells, followed by the determination of the amounts of integrin $\alpha 2$ and $\beta 1$ by ELISA. (D) Immunofluorescence images of integrin $\alpha 2 \beta 1$ (red) and lumican (green) in human fetal osteoblasts after treatment with 40 nM $6 X$ His-tagged lumican for 30 min. (E) Western blot analysis to determine the phosphorylation of ERK after pretreatment with $1 \mu \mathrm{M}$ TC-I 15 , an integrin $\alpha 2 \beta 1$ inhibitor, followed by treatment with $10 \mathrm{nM}$ lumican for $5 \mathrm{~min}$. (F) MC3T3-E1 cells were pretreated with $1 \mu \mathrm{M}$ TC-I 15, followed by treatment with the indicated concentrations of lumican in the presence of $50 \mu \mathrm{g} / \mathrm{mL}$ ascorbic acid and $10 \mathrm{mM} \beta$-glycerophosphate for 3 days, and ALP activity was assessed. (G) Western blot analysis to determine the phosphorylation of FAK and Src after pretreatment with $1 \mu \mathrm{M}$ TC-I 15, followed by treatment with 10 nM lumican for 5 min. Scale bar, $10 \mu \mathrm{m}$ (D). Data are presented as mean \pm SEM. ${ }^{*} P<0.05$ vs. control; ${ }^{\#} P<0.05$ vs. lumican-treated group. 
A

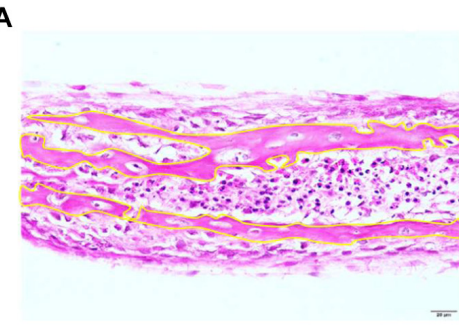

Control shRNA

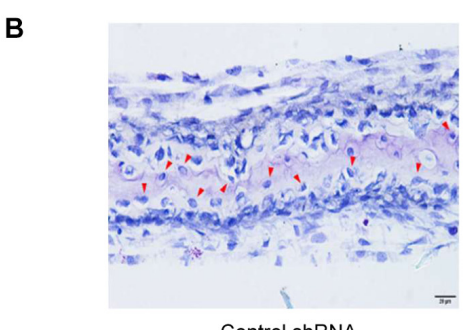

Control shRNA

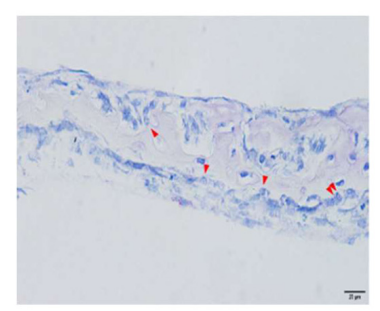

Lumican shRNA
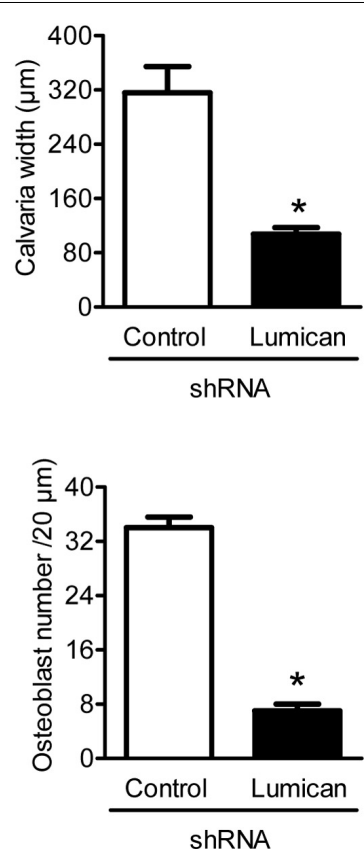

FIGURE 6 | Lumican deletion reduces calvaria bone formation. The calvaria bones dissected from timed-pregnant mice at E21.0 were infected with lumican shRNA CM in BGjb medium (containing $100 \mathrm{U} / \mathrm{mL}$ penicillin) as well as $100 \mu \mathrm{g} / \mathrm{mL}$ streptomycin and $0.1 \%$ BSA (1:1 ratio) for 3 days and additionally cultured in a medium containing $50 \mu \mathrm{g} / \mathrm{ml}$ insulin for 10 days. (A) Hematoxylin and eosin staining was performed on the specimens, and calvaria bone widths were quantitated by analyzing the average of five spots. (B) Toluidine blue $\mathrm{O}$ (blue) staining was performed on the specimens, and the number of osteoblasts (red arrows) was counted. Scale bars, $20 \mu \mathrm{m}$ (A, B). Data are presented as mean \pm SEM. ${ }^{*} P<0.05$ vs. control.

other cell types (Seomun and Joo, 2008; Brezillon et al., 2013). We thus investigated whether the protective role of lumican on osteoblasts is mediated by integrin $\alpha 2 \beta 1$. Quantitative RTPCR and western blot analyses show that integrin $\alpha 2$ and $\beta 1$ were expressed in MC3T3-E1 cells and lumican treatment augmented their levels of expression (Figures 5A,B, respectively). A binding affinity experiment using ELISA shows that the amount of lumican-associated integrin $\alpha 2$ and $\beta 1$ increased as the amount of osteoblast lysate increased (Figure 5C), indicating that lumican directly associates with integrin $\alpha 2 \beta 1$ in osteoblasts. Immunofluorescence analysis also shows the interaction between lumican and integrin $\alpha 2 \beta 1$ in osteoblasts (Figure 5D). In support of these findings, pretreatment of osteoblasts with an integrin $\alpha 2 \beta 1$ inhibitor, TC-I 15, attenuated the phosphorylation of ERK (Figure 5E), and blocked lumican-mediated stimulation of ALP activity (Figure 5F). The FAK/Src signaling is well known for the critical mediator of integrin, leading to the activation of ERK (Mitra and Schlaepfer, 2006; Bouchard et al., 2008). Importantly, pretreatment of osteoblasts with TC-I 15 reversed the lumicanmediated stimulation of FAK and Src (Figure 5G), further supporting that lumican exerts function in osteoblast via integrin $\alpha 2 \beta 1$ and downstream ERK pathway.

\section{Lumican Deletion Reduces Calvaria Bone Formation}

To confirm the importance of lumican in bone formation, we adopted the models of ex vivo explant cultures. The dissected calvaria bone from time-pregnant mice at E21.0 were infected with lumican shRNA CM, and then incubated in organ culture media. Compared to the control, calvaria thickness and osteoblast numbers were significantly decreased by the silencing of lumican (Figures 6A,B, respectively), supporting the view that lumican acts anabolically in bone metabolism.

\section{DISCUSSION}

We have shown here that lumican secreted from mature myotubes stimulates bone formation by increasing preosteoblast viability and differentiation, and these effects are likely mediated by integrin $\alpha 2 \beta 1$ and the downstream ERK signaling in osteoblasts. These findings suggest that lumican could be a myokine that, at least in part, mediates biochemical bonemuscle interactions and be a potential therapeutic target for metabolic bone diseases.

With a mass of approximately $40 \mathrm{kDa}$, lumican belongs to the family of small leucine-rich repeat proteoglycans (SLRPs; Blochberger et al., 1992). Originally identified as a major proteoglycan of the cornea (Hassell et al., 1980), lumican is now known to be widely expressed in different tissues, including skin, lung, kidney, breast, colon, artery, and cartilage (Dolhnikoff et al., 1998; Leygue et al., 1998; Schaefer et al., 2000; Nikitovic et al., 2008b). In addition to its well-known role in the regulation of the structural organization of these tissues, evidence now indicates that lumican is involved in diverse biological functions such as cell adhesion, migration, proliferation, and 
differentiation (D’Onofrio et al., 2008; Nikitovic et al., 2008a,b). Interestingly, previous proteomic analyses described lumican as a novel muscle-secreted factor (Norheim et al., 2011). The fitness benefits of the secretion of anabolic factors from muscle to bone is currently speculative, thus we tested whether lumican has protective effects in terms of bone metabolism. Our in vitro and animal experiments demonstrate that muscle-derived lumican directly stimulates osteoblastogenesis and bone formation.

Among the various transcriptional factors controlling osteogenesis, Runx2 is regarded as a master switch regulating the expression of osteoblast-specific genes including $\operatorname{Col} 1 \alpha$, Alp, and Ocn (Ducy et al., 1999). Thus, Runx2-deficient mice lack osteoblasts and fully mineralized bone matrix (Otto et al., 1997). Because of the critical role RUNX2 plays in osteoblastogenesis, this protein is tightly regulated by various cellular factors (Ge et al., 2012). In investigating the effects of lumican on RUNX2-related signals, we showed that lumican induces ERK activation in osteoblasts, which is consistent with observations in other cell types (Seomun and Joo, 2008). Moreover, ERK inhibitors attenuate lumican-stimulated Runx2 and ALP activities. Collectively, these results indicate that lumican-stimulated osteoblast differentiation may be regulated by the ERK pathway.

To determine the receptor mediating the actions of lumican on osteoblasts, we focused on integrin $\alpha 2 \beta 1$, based on the existing evidence. In particular, experimental evidence suggests that integrin $\alpha 2 \beta 1$ participates in the survival and differentiation of osteoblast lineages through the activation of ERK (Popov et al., 2011; Shih et al., 2011). In the present study, an integrin $\alpha 2 \beta 1$ inhibitor reduced lumican-induced ERK and ALP activities, suggesting that integrin $\alpha 2 \beta 1$ is the major receptor for lumican in osteoblasts.

Although we focused on the direct interaction of lumican with transmembrane integrin $\alpha 2 \beta 1$ receptor and the resultant downstream ERK activation in osteoblasts, previous study reported that U0126, the ERK inhibitor, suppressed the integrin $\beta 1$ expression induced by lumican in corneal epithelial cells (Seomun and Joo, 2008). These results suggest the other scenario where the expression of integrin $\beta 1$ is regulated by ERK phosphorylation in response to lumican. Therefore, the interplay

\section{REFERENCES}

Bettis, T., Kim, B. J., and Hamrick, M. W. (2018). Impact of muscle atrophy on bone metabolism and bone strength: implications for muscle-bone crosstalk with aging and disuse. Osteoporos. Int. 29, 1713-1720. doi: 10.1007/s00198-0184570- 1

Blochberger, T. C., Vergnes, J. P., Hempel, J., and Hassell, J. R. (1992). cDNA to chick lumican (corneal keratan sulfate proteoglycan) reveals homology to the small interstitial proteoglycan gene family and expression in muscle and intestine. J. Biol. Chem. 267, 347-352.

Bouchard, V., Harnois, C., Demers, M.-J., Thibodeau, S., Laquerre, V., Gauthier, R., et al. (2008). $\beta 1$ integrin/Fak/Src signaling in intestinal epithelial crypt cell survival: integration of complex regulatory mechanisms. Apoptosis 13, 531-542. doi: 10.1007/s10495-008-0192-y

Brezillon, S., Pietraszek, K., Maquart, F. X., and Wegrowski, Y. (2013). Lumican effects in the control of tumour progression and their links with among lumican, integrin, and ERK signal would be interesting topic to further understand the action mechanism of lumican on bone metabolism.

In summary, lumican is expressed in both the cell lysates and $\mathrm{CM}$ of myotubes. The known anabolic effects of myotube CM on bone is reduced when lumican expression in the CM is silenced. In addition, the results of additional experiments revealed that lumican could directly contribute to bone formation through the positive effects on osteoblast biology. More extensive in vivo and human studies are needed to confirm these experimental findings and determine their clinical implications.

\section{DATA AVAILABILITY STATEMENT}

The datasets used and/or analyzed during the current study are available from the corresponding author.

\section{ETHICS STATEMENT}

The animal study was reviewed and approved by the Institutional Animal Care and Use Committee of the Asan Institute for Life Sciences.

\section{AUTHOR CONTRIBUTIONS}

J-MK and B-JK: conceptualization. JL, SP, DK, and B-JK: data acquisition. JL, SP, DK, and B-JK: data analysis and interpretation. JL, SL, and B-JK: drafting of the manuscript. All authors have read and agreed to the final version of the manuscript.

\section{FUNDING}

This study was supported by the National Research Foundation of Korea (NRF) grant funded by the South Korea Government (MSIT; Grant numbers 2018R1D1A1B07045781 and 2019R1A2C2006527).

metalloproteinases and integrins. FEBS J. 280, 2369-2381. doi: 10.1111/febs. 12210

Burr, D. B., Robling, A. G., and Turner, C. H. (2002). Effects of biomechanical stress on bones in animals. Bone 30, 781-786. doi: 10.1016/s8756-3282(02)00707-x

Dolhnikoff, M., Morin, J., Roughley, P. J., and Ludwig, M. S. (1998). Expression of lumican in human lungs. Am J Respir. Cell Mol. Biol. 19, 582-587. doi: 10.1165/ajrcmb.19.4.2979

D’Onofrio, M. F., Brezillon, S., Baranek, T., Perreau, C., Roughley, P. J., Maquart, F. X., et al. (2008). Identification of betal integrin as mediator of melanoma cell adhesion to lumican. Biochem. Biophys. Res. Commun. 365, 266-272. doi: 10.1016/j.bbrc.2007.10.155

Ducy, P., Starbuck, M., Priemel, M., Shen, J., Pinero, G., Geoffroy, V., et al. (1999). A Cbfa1-dependent genetic pathway controls bone formation beyond embryonic development. Genes Dev. 13, 1025-1036. doi: 10.1101/gad.13.8.1025

Ge, C., Yang, Q., Zhao, G., Yu, H., Kirkwood, K. L., and Franceschi, R. T. (2012). Interactions between extracellular signal-regulated kinase $1 / 2$ and p38 MAP 
kinase pathways in the control of RUNX2 phosphorylation and transcriptional activity. J. Bone Miner. Res. 27, 538-551. doi: 10.1002/jbmr.561

Giudice, J., and Taylor, J. M. (2017). Muscle as a paracrine and endocrine organ. Curr. Opin. Pharmacol. 34, 49-55. doi: 10.1016/j.coph.2017.05.005

Hamrick, M. W., McNeil, P. L., and Patterson, S. L. (2010). Role of musclederived growth factors in bone formation. J. Musculoskelet. Neuronal Interact. $10,64-70$.

Harry, L. E., Sandison, A., Paleolog, E. M., Hansen, U., Pearse, M. F., and Nanchahal, J. (2008). Comparison of the healing of open tibial fractures covered with either muscle or fasciocutaneous tissue in a murine model. J. Orthop. Res. 26, 1238-1244. doi: 10.1002/jor.20649

Harry, L. E., Sandison, A., Pearse, M. F., Paleolog, E. M., and Nanchahal, J. (2009). Comparison of the vascularity of fasciocutaneous tissue and muscle for coverage of open tibial fractures. Plast. Reconstr. Surg. 124, 1211-1219. doi: 10.1097/PRS.0b013e3181b5a308

Hassell, J. R., Newsome, D. A., Krachmer, J. H., and Rodrigues, M. M. (1980). Macular corneal dystrophy: failure to synthesize a mature keratan sulfate proteoglycan. Proc. Natl. Acad. Sci. U.S.A. 77, 3705-3709. doi: 10.1073/pnas. 77.6.3705

Hirschfeld, H. P., Kinsella, R., and Duque, G. J. O. I. (2017). Osteosarcopenia: where bone, muscle, and fat collide. Osteoporos. Int. 28, 2781-2790. doi: 10. 1007/s00198-017-4151-8

Hong, A. R., and Kim, S. W. (2018). Effects of resistance exercise on bone health. Endocrinol Metab. 33, 435-444. doi: 10.3803/EnM.2018.33.4.435

Kaji, H. (2014). Interaction between Muscle and Bone. J. Bone Metab. 21, 29-40. doi: $10.11005 / \mathrm{jbm} .2014 .21 .1 .29$

Kim, B. J., Ahn, S. H., Kim, H. M., Lee, S. H., and Koh, J. M. (2015). Low skeletal muscle mass associates with low femoral neck strength, especially in older Korean women: the Fourth Korea National Health and Nutrition Examination Survey (KNHANES IV). Osteoporos. Int. 26, 737-747. doi: 10.1007/s00198-0142959-z

Kim, B. J., Lee, J. Y., Park, S. J., Lee, S. H., Kim, S. J., Yoo, H. J., et al. (2019). Elevated ceramides 18:0 and 24:1 with aging are associated with hip fracture risk through increased bone resorption. Aging (Albany NY) 11, 9388-9404. doi: 10.18632/aging.102389

Kim, B. J., Lee, Y. S., Lee, S. Y., Baek, W. Y., Choi, Y. J., Moon, S. A., et al. (2018). Osteoclast-secreted SLIT3 coordinates bone resorption and formation. J. Clin. Invest. $128,1429-1441$. doi: $10.1172 /$ jci91086

Lee, J. Y., Park, S. J., Han, S. A., Lee, S. H., Koh, J. M., Hamrick, M. W., et al. (2019). The effects of myokines on osteoclasts and osteoblasts. Biochem. Biophys. Res. Commun. 517, 749-754. doi: 10.1016/j.bbrc.2019.07.127

Lee, Y. S., Kwak, M. K., Moon, S. A., Choi, Y. J., Baek, J. E., Park, S. Y., et al. (2020). Regulation of bone metabolism by megakaryocytes in a paracrine manner. Sci. Rep. 10:2277. doi: 10.1038/s41598-020-59250-6

Leygue, E., Snell, L., Dotzlaw, H., Hole, K., Hiller-Hitchcock, T., Roughley, P. J., et al. (1998). Expression of lumican in human breast carcinoma. Cancer Res. 58, 1348-1352.

Marino, S., Staines, K. A., Brown, G., Howard-Jones, R. A., and Adamczyk, M. (2016). Models of ex vivo explant cultures: applications in bone research. Bonekey Rep. 5:818. doi: 10.1038/bonekey.2016.49

Mitra, S. K., and Schlaepfer, D. D. (2006). Integrin-regulated FAK-Src signaling in normal and cancer cells. Curr. Opin. Cell Biol. 18, 516-523. doi: 10.1016/j.ceb. 2006.08.011

Nikitovic, D., Berdiaki, A., Zafiropoulos, A., Katonis, P., Tsatsakis, A., Karamanos, N. K., et al. (2008a). Lumican expression is positively correlated with the differentiation and negatively with the growth of human osteosarcoma cells. FEBS J. 275, 350-361. doi: 10.1111/j.1742-4658.2007.06205.x

Nikitovic, D., Katonis, P., Tsatsakis, A., Karamanos, N. K., and Tzanakakis, G. N. (2008b). Lumican, a small leucine-rich proteoglycan. IUBMB Life 60, 818-823. doi: 10.1002/iub.131
Norheim, F., Raastad, T., Thiede, B., Rustan, A. C., Drevon, C. A., and Haugen, F. (2011). Proteomic identification of secreted proteins from human skeletal muscle cells and expression in response to strength training. Am. J. Physiol. Endocrinol. Metab. 301, E1013-E1021. doi: 10.1152/ajpendo.00326.2011

Otto, F., Thornell, A. P., Crompton, T., Denzel, A., Gilmour, K. C., Rosewell, I. R., et al. (1997). Cbfal, a candidate gene for cleidocranial dysplasia syndrome, is essential for osteoblast differentiation and bone development. Cell 89, 765-771. doi: 10.1016/s0092-8674(00)80259-7

Park, S. J., Lee, J. Y., Lee, S. H., Koh, J. M., and Kim, B. J. (2019). SLIT2 inhibits osteoclastogenesis and bone resorption by suppression of Cdc42 activity. Biochem. Biophys. Res. Commun. 514, 868-874. doi: 10.1016/j.bbrc.2019. 05.046

Pedersen, B. K., and Febbraio, M. A. (2012). Muscles, exercise and obesity: skeletal muscle as a secretory organ. Nat. Rev. Endocrinol. 8, 457-465. doi: 10.1038/ nrendo.2012.49

Popov, C., Radic, T., Haasters, F., Prall, W. C., Aszodi, A., Gullberg, D., et al. (2011). Integrins alpha2beta1 and alpha11beta1 regulate the survival of mesenchymal stem cells on collagen I. Cell Death Dis. 2:e186. doi: 10.1038/cddis.2011.71

Qin, Y. X., Lam, H., Ferreri, S., and Rubin, C. (2010). Dynamic skeletal muscle stimulation and its potential in bone adaptation. J. Musculoskelet. Neuronal Interact. 10, 12-24. doi: 10.1007/978-3-642-71337-8_2

Romagnoli, C., Pampaloni, B., and Brandi, M. L. (2019). Muscle endocrinology and its relation with nutrition. Aging Clin. Exp. Res. 31, 783-792. doi: 10.1007/ s40520-019-01188-5

Sabokbar, A., Millett, P. J., Myer, B., and Rushton, N. (1994). A rapid, quantitative assay for measuring alkaline phosphatase activity in osteoblastic cells in vitro. Bone Miner 27, 57-67. doi: 10.1016/s0169-6009(08)80187-0

Schaefer, L., Grone, H. J., Raslik, I., Robenek, H., Ugorcakova, J., Budny, S., et al. (2000). Small proteoglycans of normal adult human kidney: distinct expression patterns of decorin, biglycan, fibromodulin, and lumican. Kidney Int. 58, 1557-1568. doi: 10.1046/j.1523-1755.2000.00317.x

Seomun, Y., and Joo, C. K. (2008). Lumican induces human corneal epithelial cell migration and integrin expression via ERK 1/2 signaling. Biochem. Biophys. Res. Commun. 372, 221-225. doi: 10.1016/j.bbrc.2008.05.014

Shih, Y. R., Tseng, K. F., Lai, H. Y., Lin, C. H., and Lee, O. K. (2011). Matrix stiffness regulation of integrin-mediated mechanotransduction during osteogenic differentiation of human mesenchymal stem cells. J. Bone Miner Res. 26, 730-738. doi: 10.1002/jbmr.278

Tagliaferri, C., Wittrant, Y., Davicco, M. J., Walrand, S., and Coxam, V. (2015). Muscle and bone, two interconnected tissues. Ageing Res. Rev. 21, 55-70. doi: 10.1016/j.arr.2015.03.002

Verschueren, S., Gielen, E., O’Neill, T. W., Pye, S. R., Adams, J. E., Ward, K. A., et al. (2013). Sarcopenia and its relationship with bone mineral density in middle-aged and elderly European men. Osteoporos. Int. 24, 87-98. doi: 10. 1007/s00198-012-2057-z

Yu, R., Leung, J., and Woo, J. (2014). Incremental predictive value of sarcopenia for incident fracture in an elderly Chinese cohort: results from the Osteoporotic Fractures in Men (MrOs) study. J. Am. Med. Dir. Assoc. 15, 551-558. doi: 10.1016/j.jamda.2014.02.005

Conflict of Interest: The authors declare that the research was conducted in the absence of any commercial or financial relationships that could be construed as a potential conflict of interest.

Copyright $\odot 2020$ Lee, Park, Kim, Lee, Koh and Kim. This is an open-access article distributed under the terms of the Creative Commons Attribution License (CC BY). The use, distribution or reproduction in other forums is permitted, provided the original author(s) and the copyright owner(s) are credited and that the original publication in this journal is cited, in accordance with accepted academic practice. No use, distribution or reproduction is permitted which does not comply with these terms. 